\title{
Comparative Study of Complex-Ratio-Measuring Circuits and Coaxial Probe in Diagnosis of Breast Tumors
}

\author{
Nadera Najib ${ }^{1}$, Kok Yeow You ${ }^{2, *}$, Mauricio Perez ${ }^{1}$ and Robin Augustine ${ }^{1 *}$ \\ 1 Microwaves in Medical Engineering Group (MMG), Ångström Laboratory, Division of Solid State Electron- \\ ics, Department of Electrical Engineering, Uppsala University, SE-75121 Uppsala, Sweden; \\ 2 School of Electrical Engineering, Faculty of Engineering, Universiti Teknologi Malaysia; kyyou@fke.utm.my \\ * Correspondence: kyyou@fke.utm.my; Robin.Augustine@Angstrom.uu.se
}

\begin{abstract}
A developed six-port reflectometry (SPR) system was integrated to measure the relative permittivity of tumor and normal breast tissue for medical diagnostic purpose. In order to obtain an accurate and precise measurement, the calibration process was done to the SPR using the wellknown three-standard technique. Next, the studied dielectric probe was connected to the calibrated measurement-port of the SPR. The open end of the probe aperture was dibbed into the normal and tumor synthetic breast tissue samples to measure the synthetic breast tissues dielectric constant, $\varepsilon_{r}$ ', and loss factor, $\varepsilon_{r}{ }^{\prime \prime}$ in the frequency range of $1.5 \mathrm{GHz}$ to $3.3 \mathrm{GHz}$. Finally, the comparative studies were conducted between commercial VNA with Keysight $85070 \mathrm{E}$ dielectric probe and the studied SPR-probe system based on the measured magnitude of the reflection coefficient, phase shift, dielectric constant, and loss factor of the synthetic breast samples. The maximum absolute errors of the measured reflection coefficient magnitude, phase shift, dielectric constant, and loss factor were found to be $0.01,1.07^{\circ}, 1.12$, and 0.75 , respectively. It was ascertained that the predicted dielectric constant, $\varepsilon_{r}$, is able to differentiate between normal, $\left(\varepsilon_{r}{ }^{\prime}<50\right)$ and tumor, $\left(\varepsilon_{r}{ }^{\prime}>50\right)$ breast tissues.
\end{abstract}

Keywords: six-port circuits; complex-ratio-measuring circuits; open-ended coaxial probe; breast tumors; RF reflectometer; relative complex permittivity; one-port calibration; graphical user interface

\section{Introduction}

According to the World Health Organization (WHO), breast cancer is one of the leading cancers affecting women in the world. According to [1], breast tumor was the principal cause for loss of life among women (589,430 deaths) and there were over 1,658,370 new breast tumor cases in the United States alone in 2015. DePinho [2] indicated that older people are more vulnerable to getting cancer due to the accumulation of the cancer gene in their bodies over the passing years of their lives. However, the X-ray mammography has some drawbacks such as pain due to breast compression and radiation exposure. It is true that MRI is considered to be somehow costly; however, it is capable of providing more accurate diagnosis than the other detection methods [3,4]. In spite of the existence of these detection methods, they all have limitations of one kind or another. Therefore, it is very demanding to come up with a microwave technique which can detect breast cancer tumor.

Currently, there exists two applications of microwave breast imaging modalities: microwave tomography and microwave radar. The microwave tomography has the ability to create image information via measuring an array of dielectric properties at different locations of the breast. The microwave radar can plot the breast dielectric scatters but cannot create any dielectric properties image of the breast. For microwave tomography of breast imaging, the study of dielectric properties of the breast tissues is very important in order to differentiate between normal and tumor breast tissues.

An early detection of tumors signs can give a strong chance to diagnose cancer and control the disease. In order to investigate the breast tissues dielectric, a testing machine such as vector network analyzer (VNA) is required [5], However, the big size and high cost of VNA are the main drawbacks since many if not all of today's applications opt for 
compact size and low-cost measurement instruments. Due to this, this study aims to propose a six-port reflectometer (SPR) to be an alternative to the VNA.

Due to the sensitivity of the polarization of water molecules contained in the tissues, tissues show a substantial response when subjected to microwaves. In return, this enables the six-port reflectometer (SPR) sensor system to be employed as a technique to diagnose tumor in the moist tissue. The relative permittivity of the moist tissue is greatly influenced by the water volume in the moist tissue total volume because of the pure water relative permittivity.

It is well-known that the water content in the tumor tissue is relatively high compared to that of the normal tissue $(>60 \%)$ because of the increased hydration caused by the fast cancer cells metabolism of the surrounding angiogenic vasculature. It is worth mentioning that the iteration between the biological samples and the microwave relies on the complex relative permittivity values of the biological samples $\left(\varepsilon r=\varepsilon r^{\prime}-j \varepsilon r^{\prime \prime}\right)$ causing some variations in the dielectric constant, $\varepsilon r^{\prime}$ of normal $\left(\varepsilon r^{\prime}<50\right)$ and cancer tissue $\left(\varepsilon r^{\prime}>50\right)$. Therefore, it can be said that the dielectric constant can be employed to diagnose the patient's case whether has a cancer or not [6, 7]. The dielectric probe kit connected to Vector Network Analyzer (VNA) is used for microwave dielectric measurement of breast tissues and a coaxial probe kit is widely used to measure the dielectric properties of the biological tissue due to its simple, nondestructive and capable of covering a wide band operating frequency characteristic.

In this paper, a six-port reflectometer-probe system is proposed to be used for breast tumor detection instead of VNA. The integrated circuit design, dielectric measurements of synthetic breast tissues, experiment setup, and the results are presented in the following sections. In fact, microwave medical diagnostic sensor can be categorized into in vitro and in vivo measurements based on the type of tissue under test and the location of the tissues in the human body [8]. For instance, laboratory in vitro blood glucose characterization using microwave sensors were studied by [9-13]. While in vivo medical diagnostic applications using microwave sensors were investigated by [14-24]. An identification of the impact of a vein's radius using resonant microwave sensor was study based on simulation results by [14]. The microwave spiral sensor was developed by [15] for noninvasive evaluation of glycemia in animal-blood. On the other hand, several type of microwave skin patch sensors have been designed by [16-21]. The microwave sensors were also used to monitor hip fracture and intracranial pressure $[18,19]$, and measure the fluid volume changes in the heart and limb hemodynamics [20,21], respectively. In addition, non-invasive blood glucose monitors with multiple sensors have been used in clinical tests [22], while [24] proposed a set of microwave transmission sensors for real-time perception of muscle mass. Since the breast tissue used is a synthetic sample, thus the overall initial measurement in this study is an in vitro measurement.

\section{Complex-Ratio-Measuring (CRM) Circuits}

\subsection{Various Kinds of CRM Circuits}

During these fifty years, various types of complex-ratio-measuring (CRM) circuits have been studied and developed, such as three-port or four-port CRM circuits (so-called multi-probe transmission-line circuits in Figure 1 (a)) [25], five-port ring-based CRM circuits (in Figure 1(b)) [25], and six-port or seven-port CRM circuits (so-called hybrid coupler-based CRM circuits in Figure 1(c)) [25]. The three-port and four-port CRM circuits are the simplest and most economical. The disadvantage of three- or four-port circuits is that they are only suitable for use in the development of single or narrow band reflectometers.

Bandwidth of the CRM circuits can be improved by using a five-port CRM circuits (CRM circuits that have a greater number of ports). In general, the five port CRM circuits 
are built in the form of a microstrip ring line. However, the phase stability and port isolation performance for the five-port circuits are less compared to the six-port and sevenport circuits. The isolation performance and phase stability for a seven-port circuits is slightly better compared to a six-port circuits, but in terms of calibration calculation and cost, the six-port circuits are more simple and less cost than seven-port [25]. Thus, in this paper, six-port CRM circuits have been emphasized.

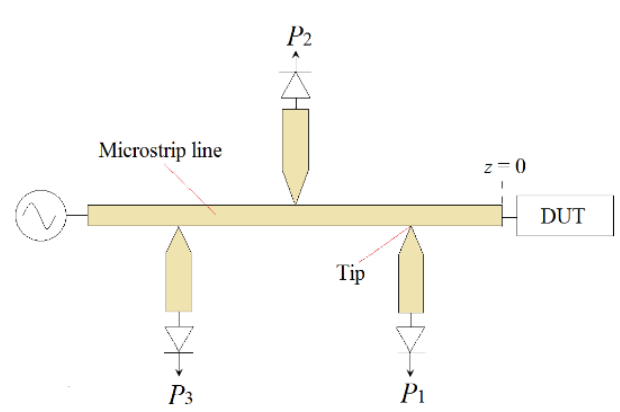

(a)

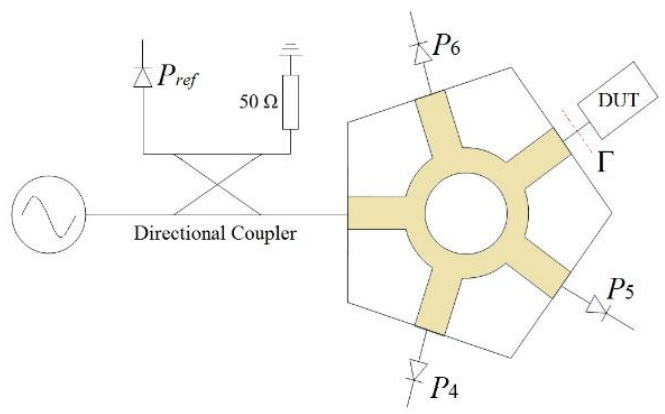

(b)

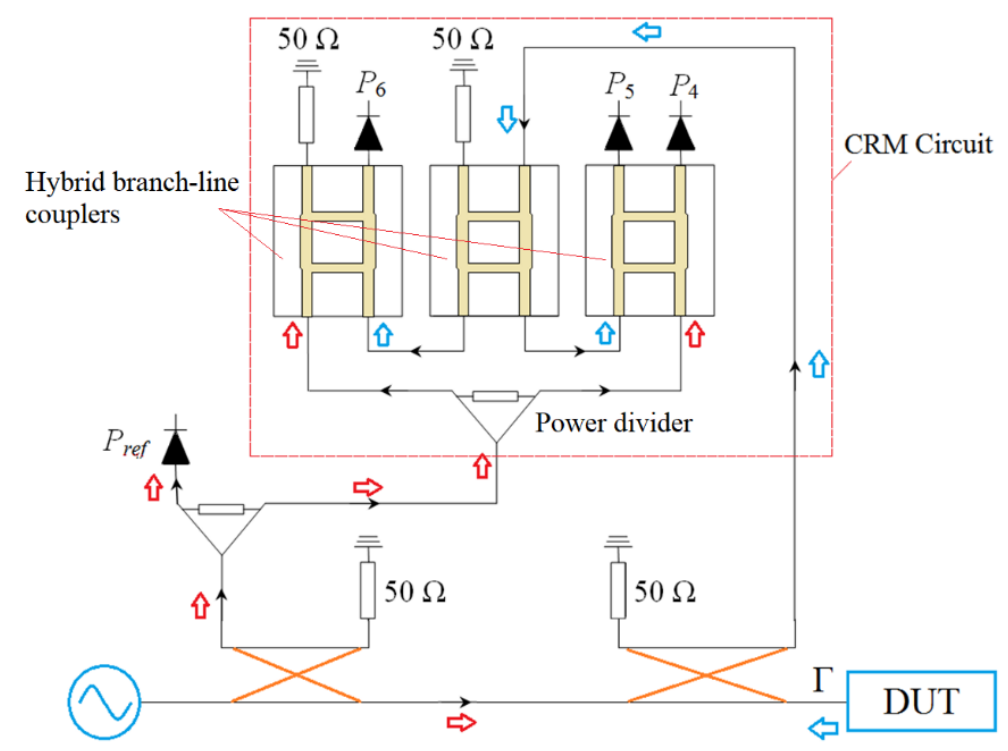

Note:

$\Rightarrow$ Incident signal $\Rightarrow$ Reflected signal

(c)

Figure 1. Typical (a) three-probe transmission-line circuit, (b) five-port ring-based CRM circuit, and (c) six-port CRM circuit [25].

\subsection{Six-Port CRM Circuits}

As mentioned, that the main components in a six-port CRM network are the three hybrid couplers and one power divider as shown in Figure 1 (c). In this study, the bandwidth and size of the CRM circuit are improved using a three-section branch-line coupler and $3 \mathrm{~dB}-W i l k i n s o n$ power divider with enhanced series-diamond stubs and meandering 
lines as shown in Figure 2 [26,27]. The modified Wilkinson power divider and the study six-port CRM circuit is capable of applying over the frequency range $1.5 \mathrm{GHz}$ to $3.3 \mathrm{GHz}$.

The integrated prototype six-port reflectometer (SPR) biosensor system is shown in Figure 3. Besides CRM circuit part, the integrated prototype system is also composed of Keysight N5171B EXG X-Series RF analog signal generator, two units of Pasternack PE2243-10 10-dB directional couplers, ICS-4749 wideband isolator, modified Wilkinson power divider, four units of Herotek DZR124AAP Schottky diodes, dielectric probe, and other digital components (four 24-bit LTC2400 ADCs, ATmega328 microcontroller, four ADA-4528 chopper amplifiers, and desktop). The complete experimental setup of the system is shown in Figure 4 (a). In addition, a MATLAB-based graphical user interface (GUI) of the reflectometer-sensor system is also developed as shown in Figure 4 (b). The detail description of the calibration routines for the study six-port CRM circuit and the aperture dielectric probe can be found in the literature [28].

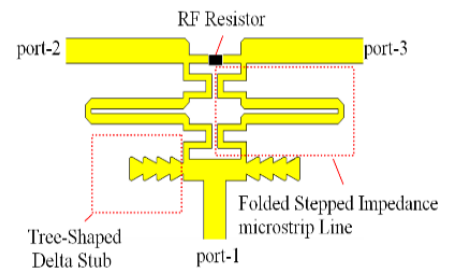

(a)

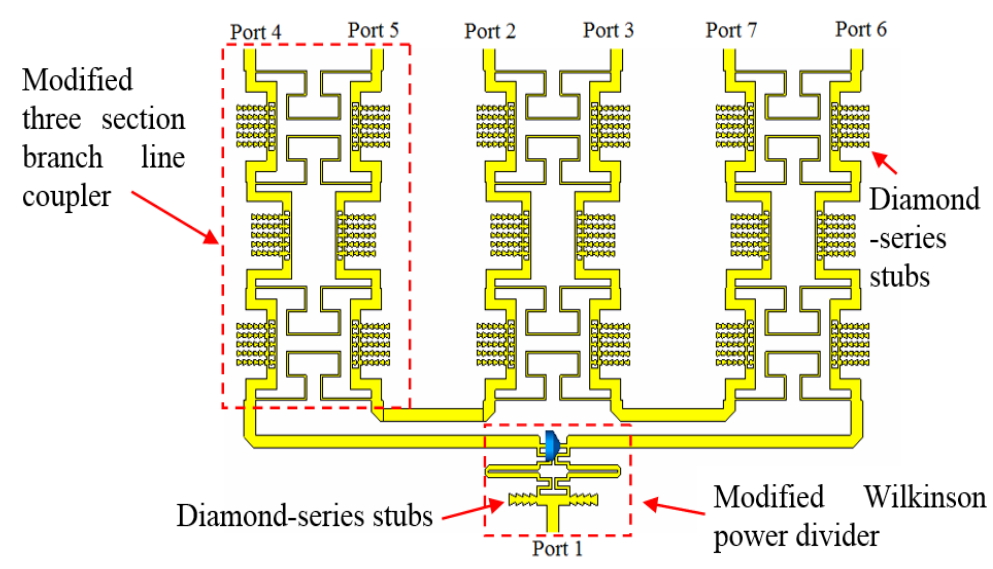

(b)

Figure 2. (a) Modified Wilkinson Power divider circuit part [26]. (b) Six-port complexratio-measuring (CRM) circuit [27]. 


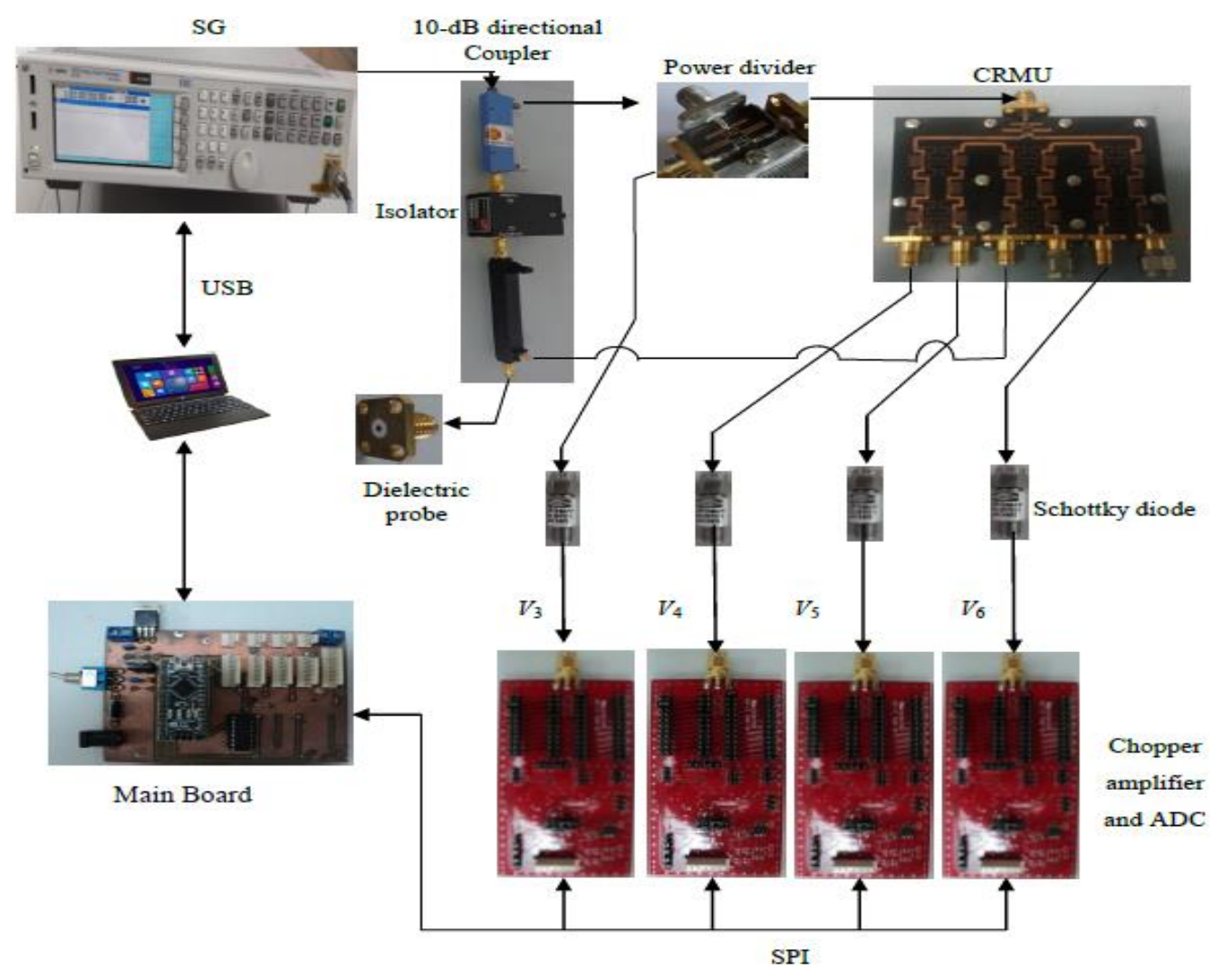

Figure 3. Six-port reflectometer (SPR) biosensor system architecture.

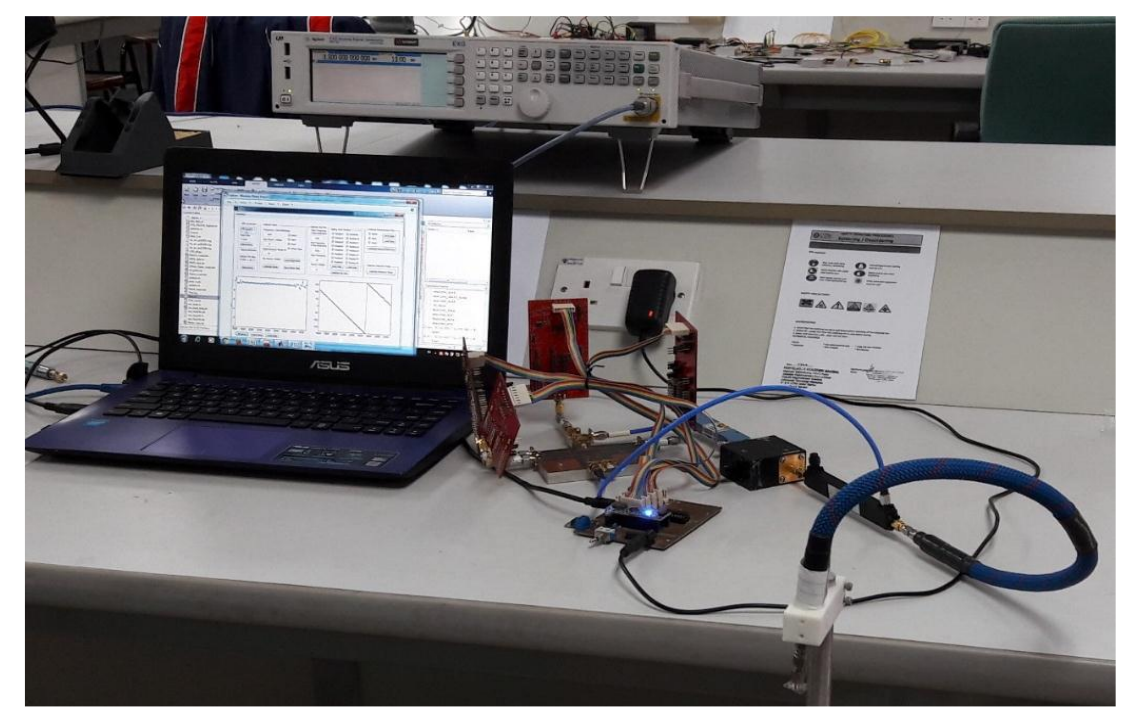

(a) 


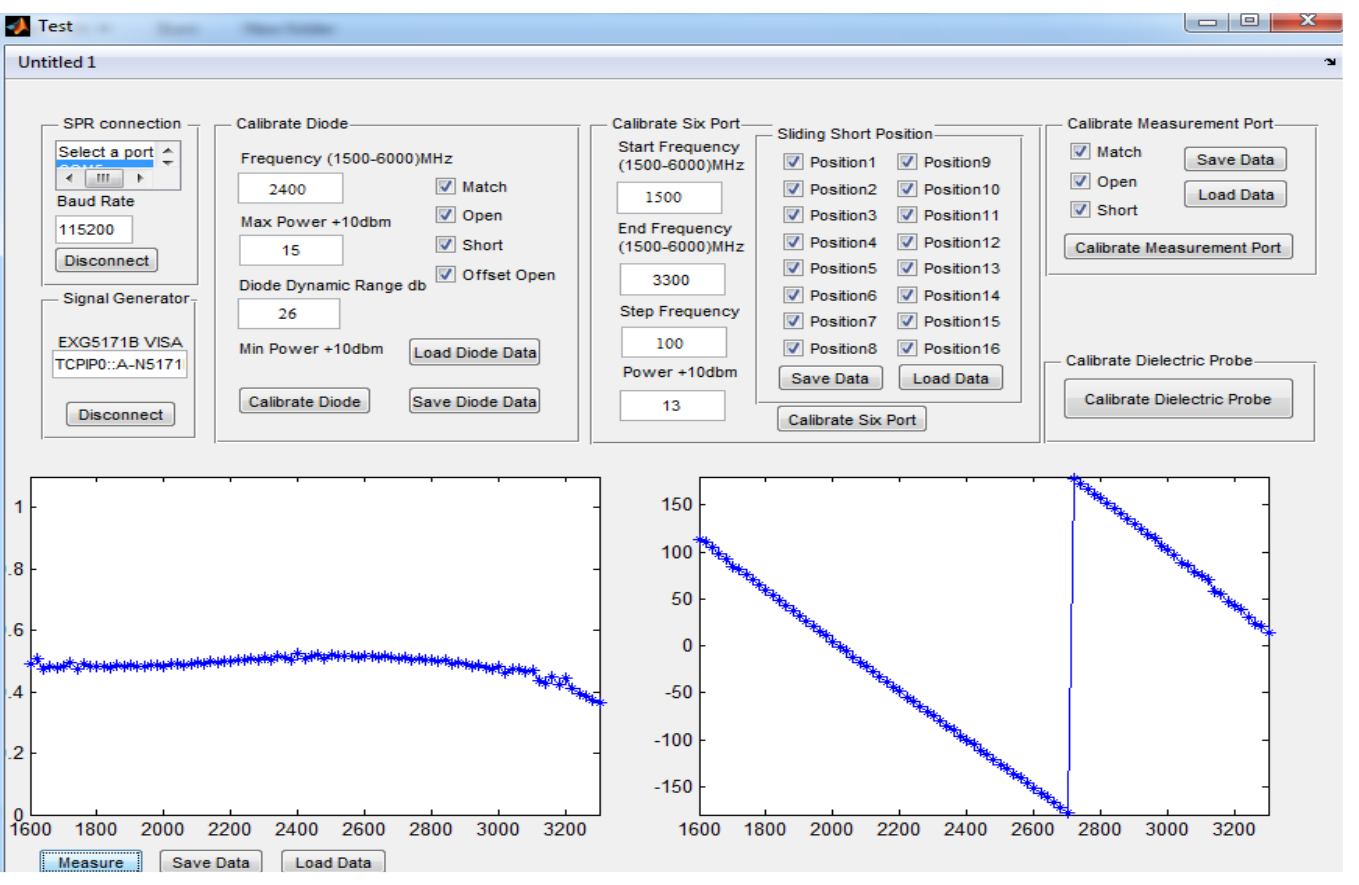

(b)

Figure 4. (a) Experimental setup. (b) Graphical user interface (GUI) for reflectometer-sensor system.

\section{Dielectric Probe}

Due to only a small volumes of the breast tissue samples required for measurement, hence the low cost SMA connector with small stub radius is constructed as dielectric probe. The stub of the SMA connector is cut and the cut surface is polished until it becomes completely even as shown in Figures 5. The dimensions of the fabricated dielectric probe have inner and outer radius conductors of $a=0.635 \mathrm{~mm}$ and $b=2.05 \mathrm{~mm}$, respectively as shown in Figure 6. The dielectric medium between inner and outer conductors is filled with Teflon with dielectric constant, $\varepsilon_{c}=2.06$ and the coaxial line length, $d$ from the connector end to the aperture probe is $7.5 \mathrm{~mm}$ as shown in Figure 6 (c). The $d$ value is very important for the conversion of the reflection coefficient at measurement port to aperture of the dielectric probe.

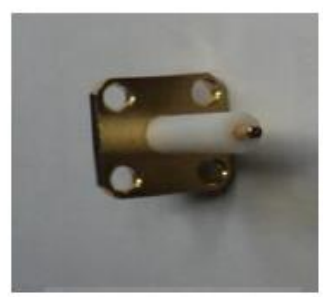

(a)

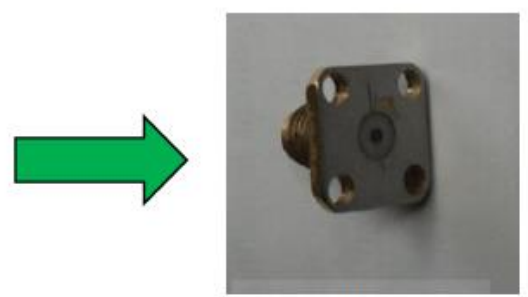

(b)

Figure 5. The photograph of (a) SMA stub panel connector (b) fabricated study dielectric probe

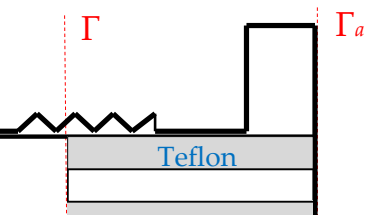




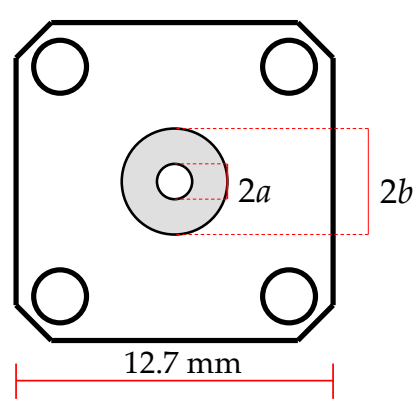

(a)

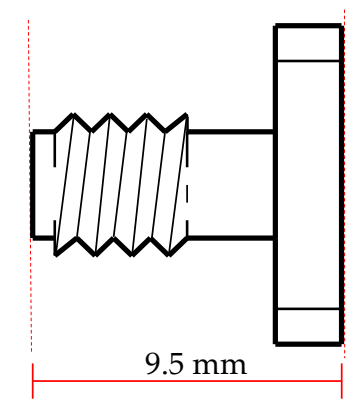

(b) (c)

Figure 6. (a) Front view, (b) side view, and (c) cross-sectional view of the fabricated dielectric probe.

\section{Synthetic Breast Tissues}

In this study, the synthetic breast samples under test are prepared using the solutions proposed by Romeo et al. [21], which is consisting of different volume ratios of water and Triton TX-100 as listed in Table 1. The certain ratio volume (based on Table 1) of the TX100 solution and water were measured using volumetric burette. Later, both of the solutions are poured and mixed into a conical flask as shown in Figure 7. Symbol $T$ denotes the solution representing the tumor breast tissues. On the other hand, the $B 1, B 2$, and $B 3$ denote the solutions for three classes of normal breast tissues, respectively. The $T$ and $B 1$ sample solutions are prepared at room temperature while the $B 2$ solution is prepared at $60^{\circ} \mathrm{C}$ because of its high viscosity and making it difficult for the stirrer to operate at room temperature.

Table 1. Synthetic breast samples consisting of different ratios of water and Triton TX-100.

\begin{tabular}{lccc}
\hline \hline \multicolumn{1}{c}{ Category } & Symbol & $\begin{array}{c}\text { TX-100 } \\
\text { (\% vol.) }\end{array}$ & $\begin{array}{c}\text { Water } \\
\text { (\% vol.) }\end{array}$ \\
\hline Tumour breast tissues & $T$ & 20 & 80 \\
Normal breast tissues (Fibroglandular tissues) & $B 1$ & 30 & 70 \\
Normal breast tissues (Fibroglandular tissues) & $B 2$ & 40 & 60 \\
Normal breast tissues (Fatty/adipose tissues) & $B 3$ & 100 & 0 \\
\hline
\end{tabular}




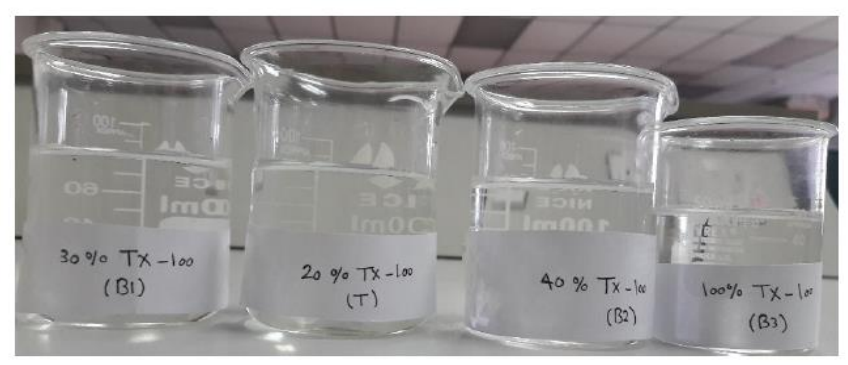

Figure 7. Prepared synthetic breast samples $T, B 1, B 2$, and $B 3$.

\section{Results and Discussion}

The performance of the integrated six-port reflectometer-biosensor system are analyzed and tested based on measured complex reflection coefficient, $\Gamma_{a}$ and predicted relative complex permittivity, $\varepsilon_{r}$ of the synthesis breast tissue. The reflection coefficient, $\Gamma_{a}$ and relative permittivity, $\varepsilon_{r}$ measurement results obtained from Keysight (formerly Agilent) E5071C network analyzer (VNA) and Keysight 85070E dielectric probe are used as a reference (as the benchmark). Furthermore, the accuracy and precision of the measurement results obtained from this study six-port reflectometer-sensor system (SPR-sensor system) and previous research five-port ring reflectometer-sensor system (FPR-sensor system) [20] are also compared.

\subsection{Reflection Coefficient}

The linear magnitude, $\left|\Gamma_{a}\right|$ and phase shift, $\phi_{a}$ of the reflection coefficient for the synthetic breast tissues $(T, B 1, B 2$, and $B 3$ ) are measured by the study SPR-sensor system and Keysight E5071C VNA with Keysight 85070E dielectric probe for comparison as shown in Figure 8 and Figure 9.

The overall measured $\left|\Gamma_{a}\right|$ (black dashed line) using SPR-sensor system seems to have ripple/noise characteristics. The ripple/noise in the measurement $\left|\Gamma_{a}\right|$ and $\phi_{a}$ are eliminated using a first-order polynomial local regression (LOESS) model with a span $=0.15$. The removed ripple $\left|\Gamma_{a}\right|$ and $\phi_{a}$ are represented by the red circled line in Figure 8 and Figure 9 , respectively. Obviously, compared with the amplitude measurement, the sensitivity of the phase shift, $\phi_{a}$ measurement of $T, B 1, B 2$, and $B 3$ is significantly higher. Therefore, the phase shift measurement can be effectively used to differentiate between the tumor and normal synthetic breast tissues samples. 


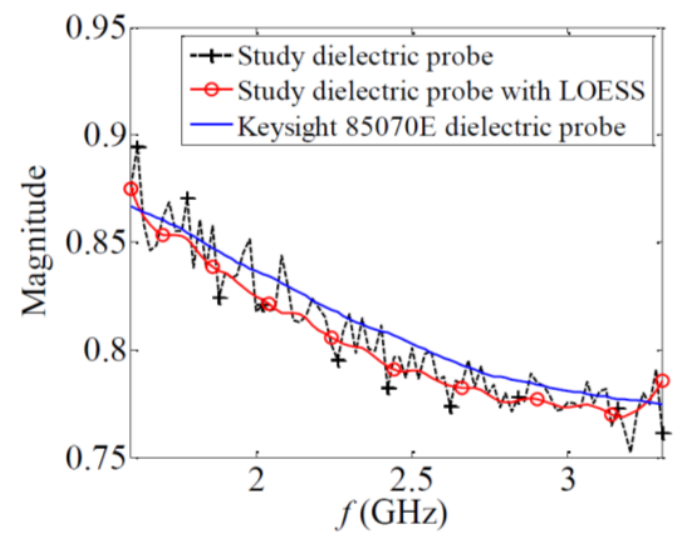

(a)

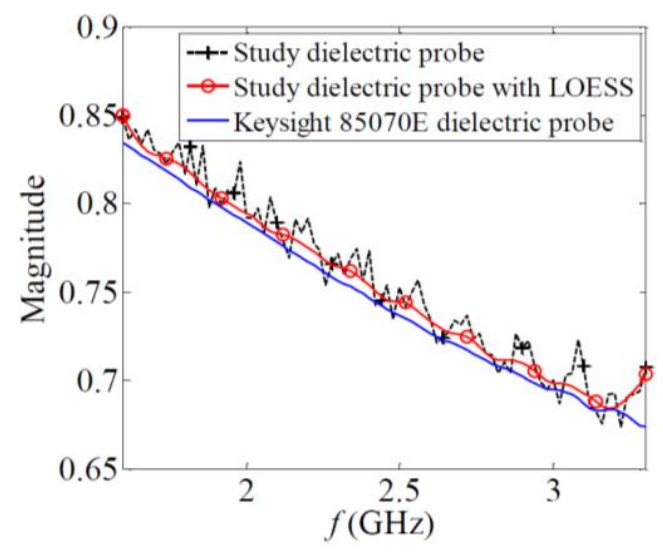

(c)

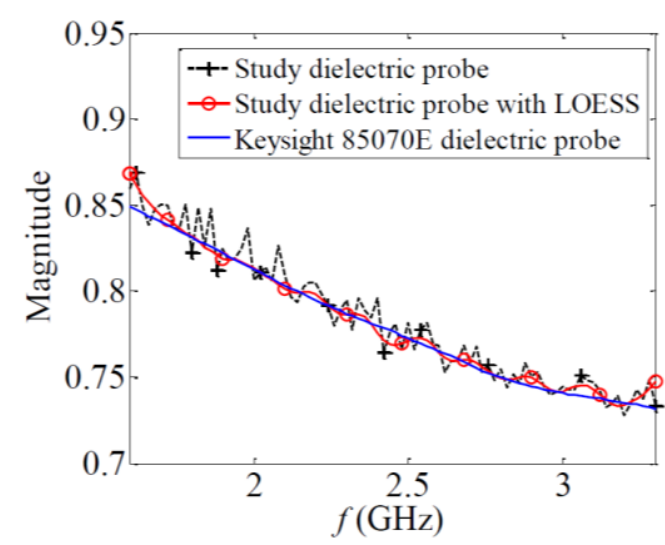

(b)

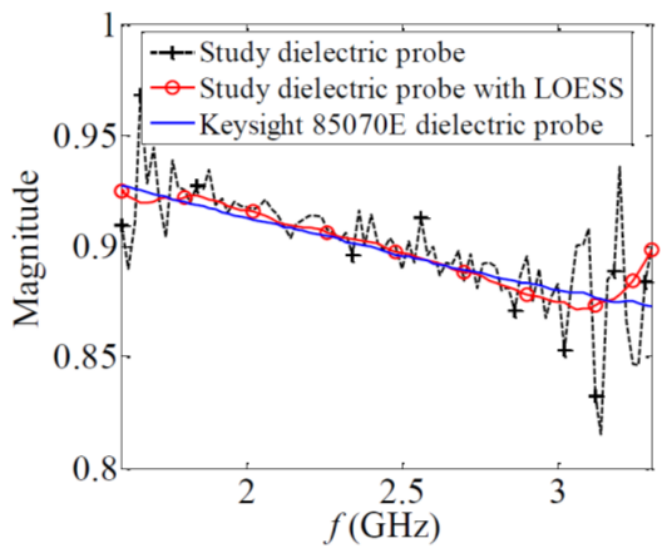

(d)

Figure 8. The measured magnitude of reflection coefficient, $\left|\Gamma_{a}\right|$ at measurement port of (a) $T$, (b) B1, (c) B2, and (d) B3, respectively

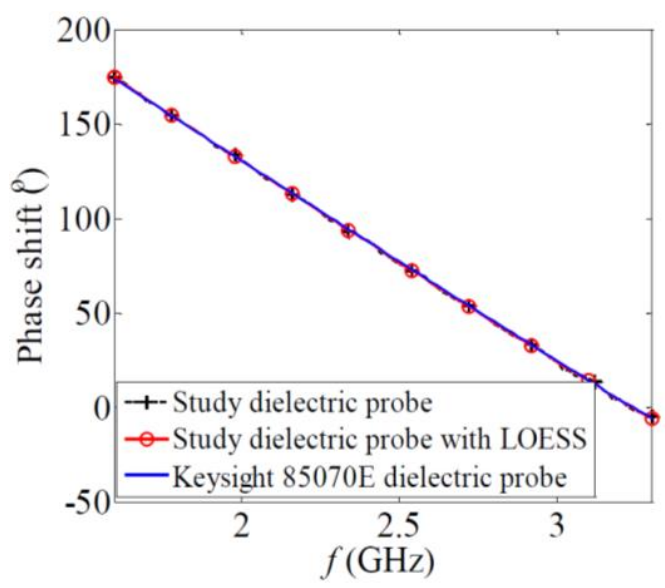

(a)

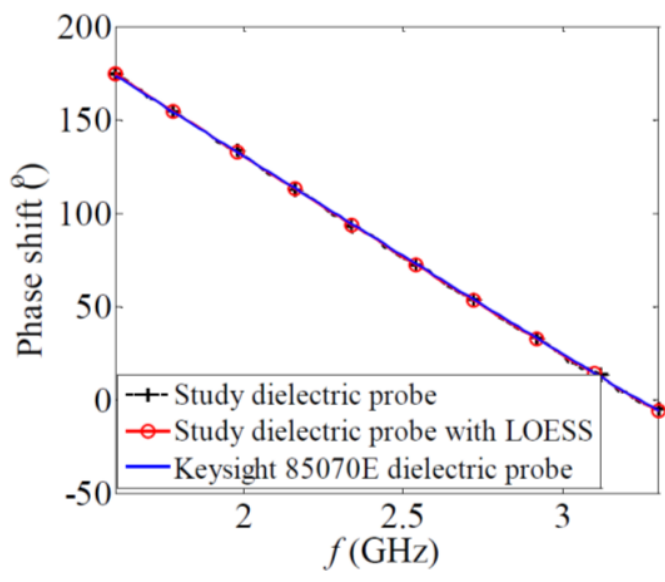

(b) 


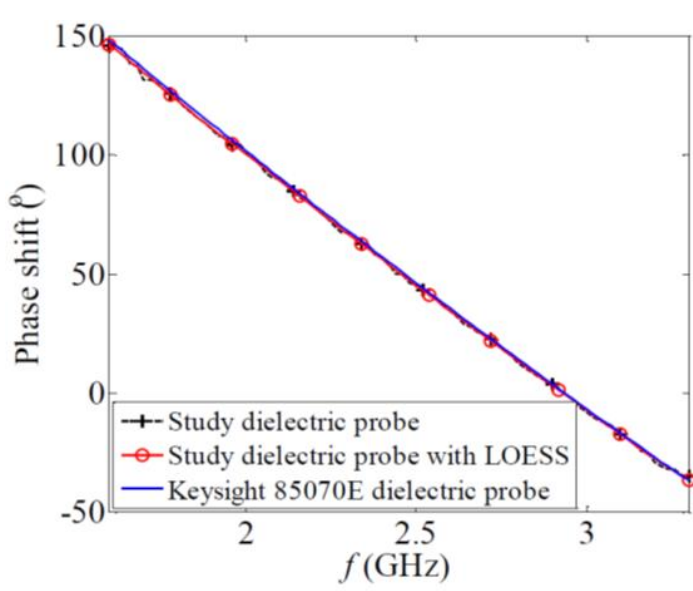

(c)

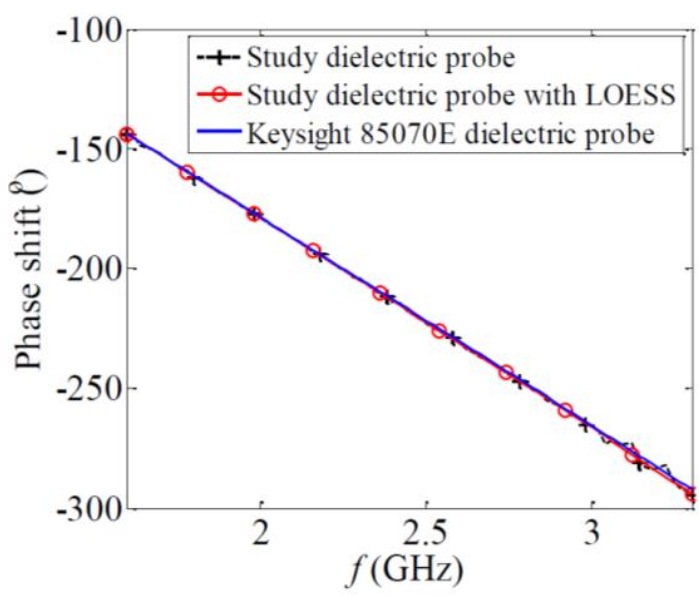

(d)

Figure 9. The measured phase shift, $\phi_{a}$ at measurement port of (a) $T$, (b) $B 1$, (c) B2, and (d) B3, respectively

The accuracy analysis of the reflection coefficient, $\Gamma_{a}$ measurement using the previous FPRsensor system [28] and the study SPR-sensor system are shown in Table 2. It should be noted that the span $=0.27$ of the local regression (LOESS) model was used by FPR-sensor system. The absolute errors of the FPR- and SPR-sensor systems in Table 2 are obtained by referring to the measurements using Keysight E5071C VNA with Keysight 85070E dielectric probe. From Table 2, the study SPR-sensor results are more accurate than FPR-sensor [28] and covered more bandwidth.

The maximum mean absolute errors of the magnitude, $\Delta\left|\Gamma_{a}\right|$ and phase, $\Delta \phi_{a}$ measurements are 0.011 and $1.22^{\circ}$, respectively, using study SPR-sensor system which are lower compared to the FPR-sensor system proposed by [28] with $\Delta\left|\Gamma_{a}\right|=0.03$ and $\Delta \phi_{a}=3.6^{\circ}$. Clearly, the error in the reflection coefficient measurement of the SPR-system is almost three times smaller than that of the FPR system [28].

Table 2. Maximum and mean absolute errors in reflection coefficient measurements.

\begin{tabular}{|c|c|c|c|c|c|c|c|c|c|}
\hline \multirow[t]{3}{*}{ Sample } & \multirow[t]{3}{*}{ LOESS } & \multicolumn{4}{|c|}{$\begin{array}{c}\text { FPR-sensor [28] } \\
(1.6 \mathrm{GHz} \text { to } 2.8 \mathrm{GHz})\end{array}$} & \multicolumn{4}{|c|}{$\begin{array}{c}\text { SPR-sensor (This study) } \\
(1.5 \mathrm{GHz} \text { to } 3.3 \mathrm{GHz})\end{array}$} \\
\hline & & \multicolumn{2}{|c|}{ Max. error } & \multicolumn{2}{|c|}{ Mean error } & \multicolumn{2}{|c|}{ Max. error } & \multicolumn{2}{|c|}{ Mean error } \\
\hline & & $\Delta\left|\Gamma_{a}\right|$ & $\Delta \phi_{a}\left(^{\circ}\right)$ & $\Delta\left|\Gamma_{a}\right|$ & $\Delta \phi_{a}\left(^{\circ}\right)$ & $\Delta\left|\Gamma_{a}\right|$ & $\Delta \phi_{a}\left(^{\circ}\right)$ & $\Delta\left|\Gamma_{a}\right|$ & $\Delta \phi_{a}\left({ }^{\circ}\right)$ \\
\hline \multirow[t]{2}{*}{$T$} & Yes & 0.04 & 5.13 & 0.01 & 1.31 & 0.015 & 1.92 & 0.0092 & 1.07 \\
\hline & No & 0.09 & 7.96 & 0.03 & 1.98 & 0.029 & 4.56 & 0.0092 & 1.22 \\
\hline \multirow[t]{2}{*}{$B 1$} & Yes & 0.04 & 4.30 & 0.01 & 1.72 & 0.014 & 1.29 & 0.0027 & 0.84 \\
\hline & No & 0.10 & 8.53 & 0.03 & 2.23 & 0.017 & 3.31 & 0.0068 & 0.96 \\
\hline \multirow[t]{2}{*}{$B 2$} & Yes & 0.04 & 3.40 & 0.01 & 0.99 & 0.029 & 0.95 & 0.0069 & 0.45 \\
\hline & No & 0.12 & 7.46 & 0.02 & 2.11 & 0.034 & 2.50 & 0.0113 & 0.78 \\
\hline \multirow[t]{2}{*}{$B 3$} & Yes & 0.04 & 4.19 & 0.02 & 3.12 & 0.026 & 1.78 & 0.0033 & 0.66 \\
\hline & No & 0.14 & 9.93 & 0.03 & 3.60 & 0.061 & 3.97 & 0.0109 & 0.85 \\
\hline
\end{tabular}


The relative complex permittivity, $\varepsilon_{r}$ values of the synthesis breast tissue are predicted from its measured complex reflection coefficient, $\Gamma$ a. The relative complex permittivity, $\varepsilon r\left(=\varepsilon r^{\prime}-j \varepsilon r^{\prime \prime}\right)$ is consisted of the real part, $\varepsilon r^{\prime}$ and imaginary part, $\varepsilon r^{\prime \prime}$, which are the dielectric constant and the loss factor, respectively.

Figures 10 and 11 show the predicted dielectric constant, $\varepsilon r^{\prime}$ and loss factor, $\varepsilon r{ }^{\prime \prime}$ values with the LOESS treated magnitude and phase of the reflection coefficient, $\Gamma_{a}$. In addition, the $\varepsilon r^{\prime}$ and $\varepsilon r "$ measurement obtained by the commercial Keysight E5071C VNA with Keysight 85070E dielectric probe is used as a benchmark for the $\varepsilon^{\prime}{ }^{\prime}$ and $\varepsilon r{ }^{\prime \prime}$ predicted by study SPR-sensor system. Although, LOESS has been applied to the raw reflection coefficient, ripple still exists in the predicted dielectric constant, $\varepsilon r^{\prime}$ and loss factor, $\varepsilon r^{\prime \prime}$. Thus, LOESS with a span $=0.15$ has been applied once to the predicted dielectric constant, $\varepsilon r^{\prime}$ and the loss factor, $\varepsilon r^{\prime \prime}$.

From Figures 10 and 11, the tumor $T$ synthetic breast tissues samples, $\varepsilon r^{\prime}$ and $\varepsilon r{ }^{\prime \prime}$ by both SPR-sensor system and VNA with Keysight 85070E dielectric probe has the highest $\varepsilon r^{\prime}$ and $\varepsilon r^{\prime \prime}$ while $B 3$ sample has the lowest $\varepsilon r^{\prime}$ and $\varepsilon r^{\prime \prime}$. The highest $\varepsilon r^{\prime}$ and $\varepsilon r{ }^{\prime \prime}$ values of the $T$ sample is due to the sample's highest water content. On the contrary, B3 possesses the lowest $\varepsilon r^{\prime}$ and $\varepsilon r^{\prime \prime}$ values due to the fact that it does not contain any water. For instance, the values of the dielectric constant, $\varepsilon^{\prime}{ }^{\prime}$ for T and B1 samples at $2.45 \mathrm{GHz}$ are in the range of 50.94 to 51.49 and 37.80 to 39.02 , respectively. It can be concluded that the $\varepsilon^{\prime}$ ' measurement can efficiently distinguish between the tumor and normal synthetic breast tissues samples, because normal $\varepsilon r^{\prime}<50$ and tumor tissue $\varepsilon r^{\prime}>50$.

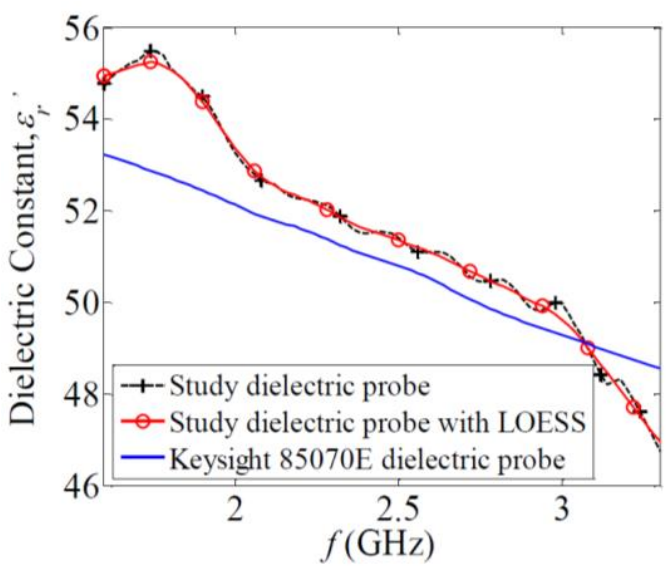

(a)

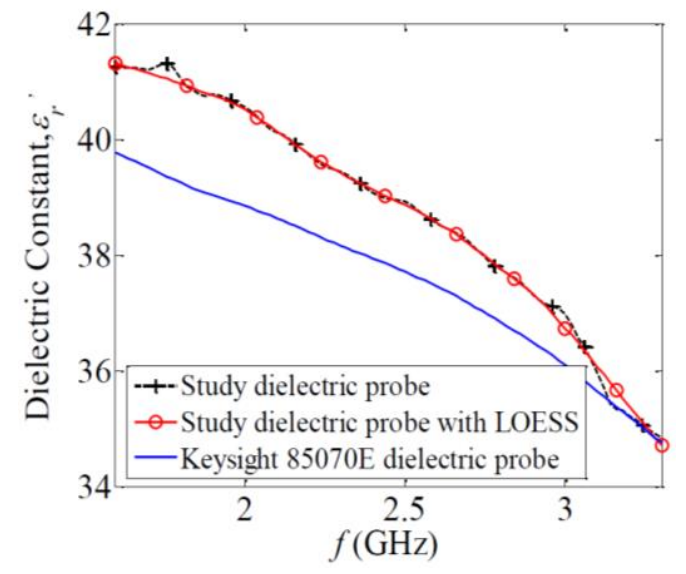

(b) 


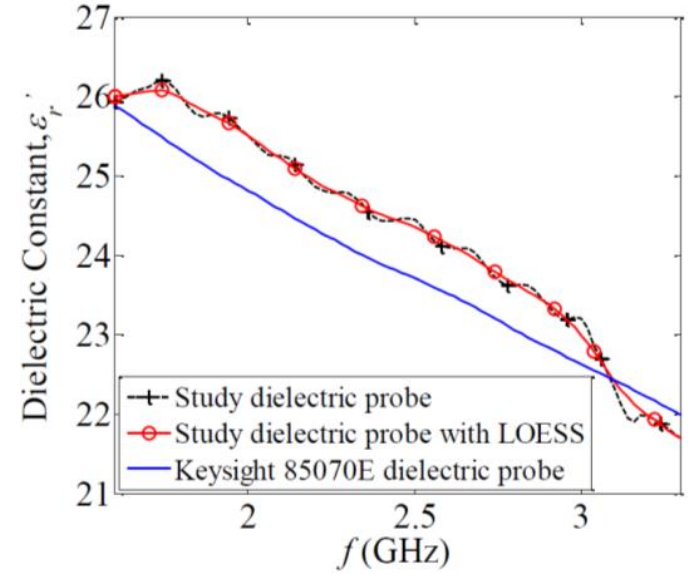

(c)

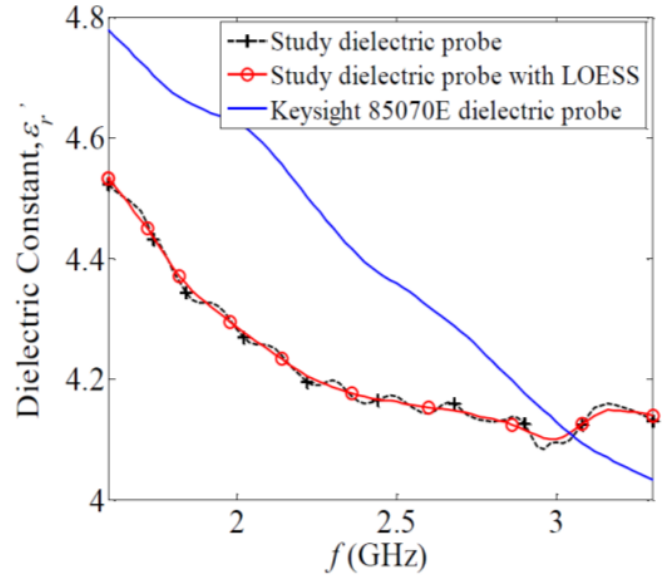

(d)

Figure 10. Predicted dielectric constant, $\varepsilon r^{\prime}$ of the synthetic breast tissues which are (a) $T$, (b) $B 1,($ c) B2, and (d) B3, respectively

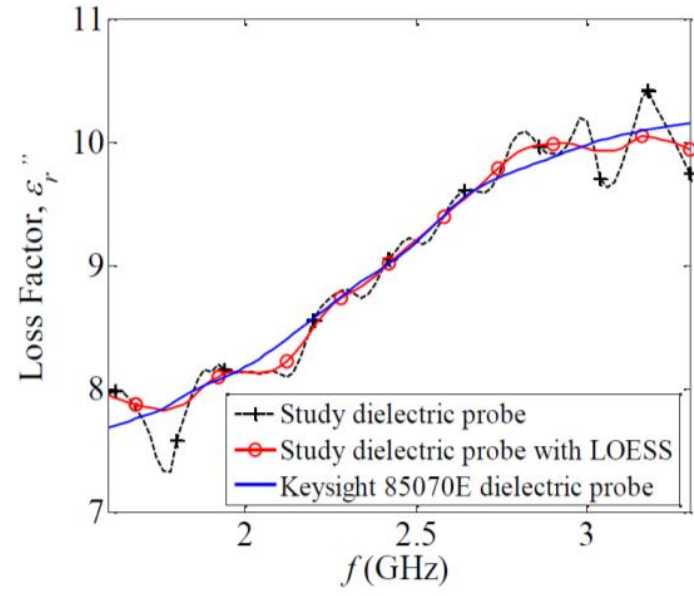

(a)

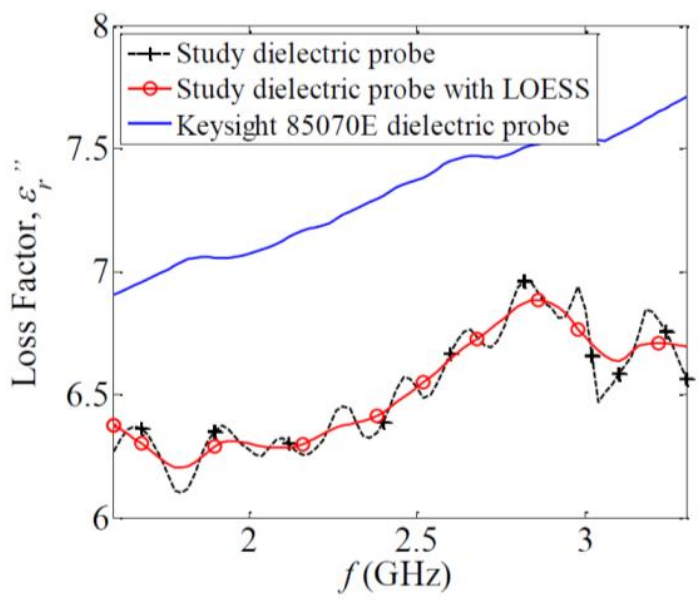

(c)

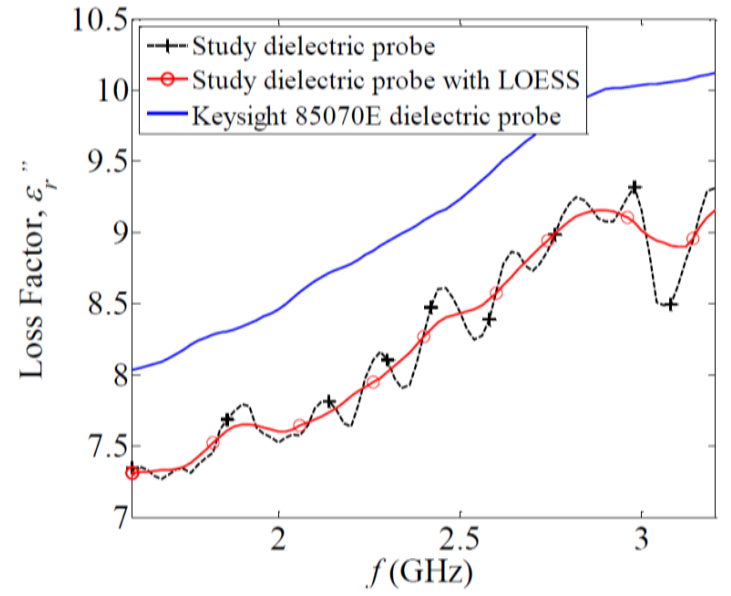

(b)

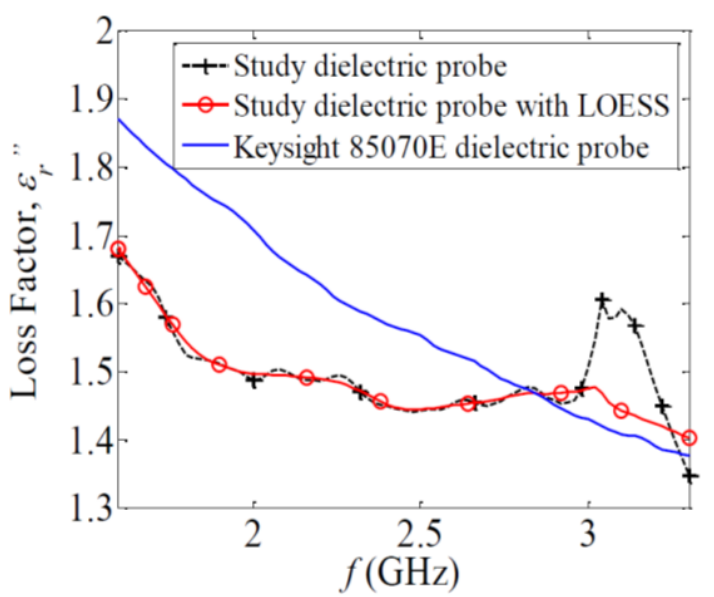

(d)

Figure 11. Predicted loss factor, $\varepsilon_{r}$ " of the synthetic breast tissues which are (a) $T$, (b) $B 1$, (c) $B 2$, and (d) B3, respectively

Table 3 present the accuracy assessment uncertainty of the dielectric constant, $\varepsilon r^{\prime}$ and loss factor, $\varepsilon r$ " of the synthetic breast with and without LOESS treatments. The maximum and mean 
errors of $\varepsilon r^{\prime}$ and $\varepsilon r r^{\prime \prime}$ measurements via full integrated SPR sensor system in this study are smaller compared to the maximum and mean proposed in [28].

Table 3. Maximum and mean absolute errors in relative complex permittivity measurements.

\begin{tabular}{|c|c|c|c|c|c|c|c|c|c|}
\hline \multirow[t]{3}{*}{ Sample } & \multirow[t]{3}{*}{ LOESS } & \multicolumn{4}{|c|}{$\begin{array}{c}\text { FPR-sensor [28] } \\
(1.6 \mathrm{GHz} \text { to } 2.8 \mathrm{GHz})\end{array}$} & \multicolumn{4}{|c|}{$\begin{array}{c}\text { SPR-sensor (This study) } \\
(1.5 \mathrm{GHz} \text { to } 3.3 \mathrm{GHz})\end{array}$} \\
\hline & & \multicolumn{2}{|c|}{ Max. error } & \multicolumn{2}{|c|}{ Mean error } & \multicolumn{2}{|c|}{ Max. error } & \multicolumn{2}{|c|}{ Mean error } \\
\hline & & $\Delta \varepsilon_{r}^{\prime}$ & $\Delta \varepsilon_{r}^{\prime \prime}$ & $\Delta \varepsilon_{r}{ }^{\prime}$ & $\Delta \varepsilon_{r}^{\prime \prime}$ & $\Delta \varepsilon_{r}{ }^{\prime}$ & $\Delta \varepsilon_{r}^{\prime \prime}$ & $\Delta \varepsilon_{r}{ }^{\prime}$ & $\Delta \varepsilon_{r}^{\prime \prime}$ \\
\hline \multirow[t]{2}{*}{$T$} & Yes & 1.98 & 2.04 & 1.55 & 1.21 & 2.21 & 0.27 & 0.88 & 0.07 \\
\hline & No & 2.76 & 2.64 & 1.61 & 1.28 & 2.43 & 0.57 & 0.92 & 0.14 \\
\hline \multirow[t]{2}{*}{$B 1$} & Yes & 0.77 & 1.61 & 0.38 & 1.10 & 1.73 & 0.93 & 1.19 & 0.75 \\
\hline & No & 1.65 & 2.45 & 0.56 & 1.14 & 1.95 & 1.16 & 1.30 & 0.75 \\
\hline \multirow[t]{2}{*}{$B 2$} & Yes & 1.63 & 1.51 & 1.02 & 0.96 & 0.71 & 1.04 & 0.53 & 0.75 \\
\hline & No & 2.08 & 1.97 & 1.03 & 0.95 & 0.79 & 1.20 & 0.54 & 0.75 \\
\hline \multirow[t]{2}{*}{ B3 } & Yes & 2.13 & 0.59 & 1.87 & 0.27 & 0.34 & 0.24 & 0.20 & 0.11 \\
\hline & No & 2.38 & 0.93 & 1.85 & 0.35 & 0.38 & 0.26 & 0.20 & 0.13 \\
\hline
\end{tabular}

\section{Conclusions}

The SPR sensor system was developed for reflection coefficient measurement and the measurement was benchmarked with Keysight E5071C VNA with Keysight 85070E dielectric using the microwave (ISM-band) frequency. The synthetic breast tissues were obtained using various mixture ratios of Triton TX-100 and water to mimic the conductivity and dielectric of the human breast tissues. The integrated SPR-sensor system performance assessment was investigated via the measurement of the complex reflection coefficient (magnitude and phase) and the relative complex permittivity (dielectric constant, $\varepsilon r^{\prime}$ and loss factor, $\varepsilon r^{\prime \prime}$ ) of synthetic breast tissues.

Due to the appearance of ripples in the magnitude and phase shift measurements by SPR, the LOESS model is applied to smoothen the curves of these measurements. In addition, both predicted dielectric constant, $\varepsilon r^{\prime}$ and loss factor, $\varepsilon r$ " from the LOESS treated magnitude and phase shift curves seem to have ripple characteristics as well. Therefore, the LOESS is again applied to the predicted $\varepsilon r^{\prime}$ and $\varepsilon r^{\prime \prime}$ measurements to smoothen their curves. In sum, the predicted dielectric constant, $\varepsilon r^{\prime}$ has the potential to differentiate between normal and tumor breast tissues. While the loss factor, $\varepsilon_{r}$ " measurement cannot be used as an alternative to differentiate between the tumor and normal synthetic breast tissues samples.

Author Contributions: Conceptualization, K.Y.Y. and N.N.; methodology, K.Y.Y., N.N., R.A., and M.P.; software, K.Y.Y. and N.N.; validation, R.A., K.Y.Y., M.P.; formal analysis, K.Y.Y., N.N, R.A. and M.P.; investigation, N.N., K.Y.Y, R.A., and M.P; resources, K.Y.Y., N.N. and R.A.; data curation, N.N. and K.Y.Y.; writing-original draft preparation, N.N., K.Y.Y., R.A. and M.P.; writing-review and editing, N.N., K.Y.Y., R.A. and M.P.; visualization, N.N., K.Y.Y., R.A., and M.P.; supervision, K.Y.Y., R.A. and M.P.; project administration, R.A., K.Y.Y. and M.P.; funding acquisition, K.Y.Y. and R.A.

Funding: The article is partially funded by Eurostars MAS (E! 114232).

Acknowledgments: The authors would like to thank the financial support of the Swedish Foundation for Strate-gic Research projects LifeSec (RIT17-0020) and Zero IoT (CHI19-0003). This study was supported by Universiti Teknologi Malaysia and Uppsala University under project number thors also would like to thank to Chia-Yew Lee for their invaluable support Au- 
Conflicts of Interest: The authors declare no conflict of interest.

\section{References}

1. Torre, L. A., Bray, F., Siegel, R. L., Ferlay, J., Lortet-Tieulent, J. and Jemal, A. Global Cancer Statistics, 2012. CA: a Cancer Journal for Clinicians. 2015. 65: 87-108.

2. DePinho, R. A. The Age of Cancer. Nature. 2000. 408: 248-254

3. Verbelen, Hanne, et al. Breast edema, from diagnosis to treatment: state of the art.Archives of physiotherapy. 2021, 11.1, 1-10.

4. Johansson K, Jönsson C, Björk-Eriksson T. Compression treatment of breast edema: a randomized controlled pilot study. Lymphat Res Biol. 2020, 18(2), 129-35.

5. Simonov, N., Jeon, S.-I., Kim, B.-R., Lee, K.-J. and Son, S.-H. Acceleration of Electromagnetic Solver for Microwave Tomography. Proceedings of the URSI Asia- Pacific Radio Science Conference (URSI AP-RASC): Aug 21 IEEE. 2016. 590-593.

6. Champbell AM, Land DV. Dielectric Properties of Female Human Breast Tissue Measured In Vitro at 3.2 GHz. Phys Med Biol. 1992. 37:193-210.

7. Cheng, Yiou, and Minghuan Fu. Dielectric properties for non-invasive detection of normal, benign, and malignant breast tissues using microwave theories. Thoracic cancer 2018, 9.4, 459-465.

8. Jiang, Cheng, et al. Antifouling strategies for selective in vitro and in vivo sensing. Chemical reviews. 2020. $120: 3852-3889$.

9. Jha, A.K.; Akhter, Z.; Tiwari, N.; Shafi, K.T.M.; Samant, H.; Akhtar, M.J.; Cifra, M. Broadband wireless sensing system fornoninvasive testing of biological samples. IEEE J. Emerg. Sel. Top. Circuits Syst. 2018, 8, 251-259.

10. Vrba, J.; Karch, J.; Vrba, D. Phantoms for development of microwave sensors for noninvasive blood glucose monitoring. Int. J. Antennas Propag. 2015, 2015, 570870.

11. Kim, J.; Babajanyan, A.; Hovsepyan, A.; Lee, K.; Friedman, B. Microwave dielectric resonator biosensor for aqueous glucose solution. Rev. Sci. Instrum. 2008, 79, 086107.

12. . Choi, H.; Naylon, J.; Luzio, S.; Beutler, J.; Birchall, J.; Martin, C.; Porch, A. Design and in vitro interference test of microwave noninvasive blood glucose monitoring sensor. IEEE Trans. Microw. Theory Tech. 2015, 63, 3016-3025.

13. Sen K, Anand S. Demonstration of microstrip sensor for the feasibility study of non-invasive blood-glucose sensing. Mapan. 2021 Mar;36(1):193-9.

14. .Babajanyan A, Minasyan B, Odabashyan L, Kim S, Kim J, Lee JH, Friedman B, Lee K. Noninvasive in Vivo Evaluation of Mouse-Blood Glycemia with a Microwave Spiral Sensor. Journal of Contemporary Physics (Armenian Academy of Sciences). 2021 Jan;56(1):47-54.

15. Zidane MA, Amar H, Rouane A. Study of Two Constraints Impacting Measurements of Human Glycemia Using a Microwave Sensor. Biosensors. 2021 Mar;11(3):83.

16. Perez, M.D.; Jeong, S.H.; Raman, S.; Nowinski, D.; Wu, Z.; Redzwan, S.M.S.; Velander, J.; Peng, Z.; Hjort, K.; Augustine, R.Headcompliant microstrip split ring resonator for non-invasive healing monitoring after craniosynostosis-based surgery. Healthc. Technol. Lett. 2020, 7, 29-34

17. Mohd Shah, S.R.; Asan, N.B.; Velander, J.; Ebrahimizadeh, J.; Perez, M.D.; Mattsson, V.; Blokhuis, T.; Augustine, R. Analysis of thickness variation in biological tissues using microwave sensors for health monitoring applications. IEEE Access 2019, 7, 156033-156043

18. Perez, M.D.; Redzwan Mohd Shah, S.; Velander, J.; Raaben, M.; Badariah Asan, N.; Blokhuis, T.; Augustine, R. Microwave sensors for new approach in monitoring hip fracture healing. In Proceedings of the 2017 11th European Conference on Antennas and Propagation (EUCAP), Paris, France, 19-24 March 2017; pp. 1838-1842.

19. Griffith, J.; Cluff, K.; Eckerman, B.; Aldrich, J.; Becker, R.; Moore-Jansen, P.; Patterson, J. Non-invasive electromagnetic skin patch sensor to measure intracranial fluid-volume shifts. Sensors 2018, 18, 1022.

20. Alruwaili, F.; Cluff, K.; Griffith, J.; Farhoud, H. Passive self resonant skin patch sensor to monitor cardiac intraventricular stroke volume using electromagnetic properties of blood. IEEE J. Transl. Eng. Heal. Med. 2018, 6, 1-9.

21. Cluff, K.; Patterson, J.; Becker, R.; Jayakumar, B.; Han, K.; Condon, E.; Dudley, K.; Szatkowski, G.; Pipinos, I.I.; Amick, R.Z. Passive wearable skin patch sensor measures limb hemodynamics based on electromagnetic resonance. IEEE Trans. Biomed. Eng. 2018, 65, 847-856.

22. Feng R, Geng Z, Li A, Chu Y, Wu C, Zhang N, Guo X, Zhang J, Wang X, Tang F. Noninvasive blood glucose monitor via multi-sensor fusion and its clinical evaluation. Sensors and Actuators B: Chemical. 2021 Apr 1;332:129445.

23. Shah, S.R.M.; Velander, J.; Mathur, P.; Perez, M.D.; Asan, N.B.; Kurup, D.G.; Blokhuis, T.J.; Augustine, R. Split-ring resonator sensor penetration depth assessment using in vivo microwave reflectivity and ultrasound measurements for lower extremity trauma rehabilitation. Sensors 2018, 18, 636.

24. Mattsson, V.; Ackermans, L.L.G.C.; Mandal, B.; Perez, M.D.; Vesseur, M.A.M.; Meaney, P.; Ten Bosch, J.A.; Blokhuis, T.J.; Augustine, R. MAS: Standalone Microwave Resonator to Assess Muscle Quality. Sensors 2021, 21, 5485. 
25. You, K. Y., Nadera N, Lee, K. Y., Cheng, E. M., Lee, Y. S. 2019. Chapter 3 Microwave Complex-Ratio-Measuring Circuits: Alternative Solutions to Microwave Vector Instruments. In: Handbook of Research on Recent Developments in Electrical and Mechanical Engineering. Edit by Jamal Zbitou, Catalin Iulian Pruncu, and Ahmed Errkik. 87-122. IGI Global. ISBN No: 9781799801177.

26. Nadera, N., You, K. Y., Mohamad Ngasri, D., Nor Hisham, K. Wideband and compact wilkinson power divider utilizing series delta-stub and folded stepped-impedance transmission line, Radioengineering. 2018. 27:200-206.

27. Nadera, N. and You, K.Y., Broadband and compact complex ratio measuring unit. Microwave and Optical Technology Letters. 2018. 60:3039-3045.

28. Lee, C. Y., You, K. Y., Abbas, Z., Lee, K. Y., Lee, Y. S. and Cheng, E. M. S-band Five-Port Ring Reflectometer-Probe System for in Vitro Breast Tumor Detection. International Journal of RF and Microwave Computer-Aided Engineering. 2018. 28. e21198

29. Romeo, S., Di Donato, L., Bucci, O. M., Catapano, I., Crocco, L., Scarfi, M. R. and Massa, R. Dielectric Characterization Study of Liquid-Based Materials for Mimicking Breast Tissues. Microwave and Optical Technology Letters. 2011. 53: 1276-1280. 